\title{
Adomian Decomposition Method for a Nonlinear Heat Equation with Temperature Dependent Thermal Properties
}

\author{
Ashfaque H. Bokhari, ${ }^{1}$ Ghulam Mohammad, ${ }^{2}$ M. T. Mustafa, $^{1}$ \\ and F. D. Zaman ${ }^{1}$ \\ ${ }^{1}$ Department of Mathematics and Statistics, King Fahd University of Petroleum and Minerals, Dhahran \\ 31261, Saudi Arabia \\ ${ }^{2}$ Department of Mathematics, National College of Business Administration and Economics, Lahore, \\ Pakistan
}

Correspondence should be addressed to M. T. Mustafa, tmustafa@kfupm.edu.sa

Received 26 December 2008; Revised 20 April 2009; Accepted 14 July 2009

Recommended by Saad A. Ragab

The solutions of nonlinear heat equation with temperature dependent diffusivity are investigated using the modified Adomian decomposition method. Analysis of the method and examples are given to show that the Adomian series solution gives an excellent approximation to the exact solution. This accuracy can be increased by increasing the number of terms in the series expansion. The Adomian solutions are presented in some situations of interest.

Copyright (C) 2009 Ashfaque H. Bokhari et al. This is an open access article distributed under the Creative Commons Attribution License, which permits unrestricted use, distribution, and reproduction in any medium, provided the original work is properly cited.

\section{Introduction}

In the classical model of the heat equation, the thermal diffusivity and thermal conductivity of the medium are assumed to be constant. In some media such as gases, these parameters are proportional to the temperature of the medium giving rise to a nonlinear heat equation of the following form [1]:

$$
C(x) \frac{\partial u}{\partial t}=\lambda \frac{\partial}{\partial x}\left(k u \frac{\partial u}{\partial x}\right)
$$

where $C$ is the conductivity, $k$ is diffusivity, and $\lambda$ is a constant. 
However, in some situations the diffusivity is proportional to $u^{\alpha}$, which gives rise to a more general nonlinear heat equation

$$
C(x) \frac{\partial u}{\partial t}=\lambda \frac{\partial}{\partial x}\left(u^{\alpha} \frac{\partial u}{\partial x}\right)
$$

In this paper we investigate the nonlinear heat equation

$$
\frac{\partial u}{\partial t}=\frac{\partial}{\partial x}\left(f(u) \frac{\partial u}{\partial x}\right)
$$

with $f(u)=u^{m}$, using the Adomian decomposition method. This method was presented by Adomian to solve algebraic, differential, integrodifferential equations and stochastic problems [2-5]. In these papers Adomian presented the so-called decomposition method in which the problem is split into linear (solvable) and nonlinear part. By assuming that the solution admits a power series representation, the nonlinear contribution to the solution is obtained in the form of "Adomian polynomials" [6]. Alternative methods of calculating Adomian polynomials have been discussed by Babolian and Javadi [7] and Wazwaz [811]. For the convergence of the Adomian method, see [12-14]. For a detailed treatment and applications of the Adomian decomposition method one may refer to [6]. Chiu and Chen [15] have applied the Adomian method to study fin problem with variable conductivity. Wazwaz in [10] established an algorithm for calculating Adomian polynomials that depend mainly on algebraic and trigonometric identities and on Taylor's expansion. A feature of this method is that it involves less formulas and is straightforward to implement. The reader is referred to [10, Section 2] for details of algorithm and its connection with earlier approach of Adomian [6]. We will use the modified Adomian algorithm given by Wazwaz [10] to find the Adomian solutions to our models of nonlinear heat equation with temperature dependent diffusivity.

\section{Method of Solution}

Introducing the operator $L_{t}=\partial / \partial t,(1.3)$ takes the form

$$
L_{t} u(x, t)=\left[f^{\prime}(u) u_{x}^{2}+f(u) u_{x x}\right]
$$

We solve (2.1) subject to the initial condition

$$
u(x, 0)=g(x)
$$

Applying inverse operator $L_{t}^{-1}$ to both sides of (2.1) yields

$$
u(x, t)=u(x, 0)+L_{t}^{-1}\left[\left(f^{\prime}(u) u_{x}^{2}+f(u) u_{x x}\right)\right]
$$


The desired series solution by Adomian decomposition method is given by (cf. [2-6] for details)

$$
u(x, t)=\sum_{n=0}^{\infty} u_{n}(x, t)
$$

and $u_{1}, u_{2}, u_{3}, \ldots$ are calculated from recursive relation

$$
\begin{aligned}
u_{0} & =u(x, 0), \\
u_{n+1} & =L_{t}^{-1}\left[\left(A_{n}\right)\right], \quad n \geq 0,
\end{aligned}
$$

where $A_{n}$ are the Adomian polynomials for the nonlinear operator

$$
F(u(x, t))=f^{\prime}(u) u_{x}^{2}+f(u) u_{x x}
$$

The formulas that can be used to generate Adomian polynomials are discussed by Adomian in [6]. Here we employ the algorithm of Wazwaz [10] to calculate Adomian polynomials, which seems quite natural and suited for implementation by software.

\section{Applications and Results}

We consider the nonlinear heat equation

$$
\begin{gathered}
\frac{\partial u}{\partial t}=\frac{\partial}{\partial x}\left(f(u) \frac{\partial u}{\partial x}\right), \\
u(x, 0)=g(x)
\end{gathered}
$$

with power nonlinearity $f(u)=u^{m}$. We are interested in investigating the case of power nonlinearity due to the fact that this assumption is made in most of the applied nonlinear problems of heat transfer and flows in porous media. For instance, $f(u)=u^{-1 / 2}$ corresponds to fast diffusion processes of plasma diffusion and thermal expulsion of liquid Helium [1618]. The diffusivity $f(u)=u^{2}$ is used to model process of melting and evaporation of metals [17-19]. For the initial temperature profile, we consider typical cases like $g(x)$ a quadratic function or $g(x)=e^{-a x^{2}}$ or $g(x)=\operatorname{sech}^{2} x$ which corresponds to soliton like initial profile.

Case $A\left(g(x)=a x^{2}+b x+c\right)$. The Adomian solution $u(x, t)$ for general $a, b, c$, and $m$ can be obtained from authors as Mathematica file. Some particular cases for $a, b, c$, and $m$ are considered as follows.

(i) $a=b=c=1$ and $m=2$. 
The Mathematica code to obtain Adomina solution in this case consists of the following commands:

$$
\begin{aligned}
& f\left[n_{-}\right]=\sum_{i=0}^{n-1} u_{i}[x] \alpha^{i}+O[\alpha]^{n}, \\
& f 1 d\left[n_{-}\right]=\sum_{i=0}^{n-1} \partial_{x} u_{i}[x] \alpha^{i}+O[\alpha]^{n}, \\
& f 2 d\left[n_{-}\right]=\sum_{i=0}^{n-1} \partial_{x, x} u_{i}[x] \alpha^{i}+O[\alpha]^{n},
\end{aligned}
$$

maximum number of polynomials and solution terms:

$$
k=5
$$

Finding Adomian polynomials:

$$
\begin{aligned}
& \text { apoly }=m f[k]^{m-1} f 1 d[k]^{2} / / \text { Simplify } \\
& \text { bpolynomial }=f[k]^{m} f 2 d[k] .
\end{aligned}
$$

Making vector of admian polynomials:

$$
v=\text { CoefficientList }[\text { coeffpoly }, \alpha] \text {. }
$$

Finding solution $u(x, t)$ :

$$
\begin{gathered}
u_{0}\left[x_{-}\right]=a * x^{2}+b * x+c \\
\operatorname{Do}\left[u_{i}\left[x_{-}\right]=\int_{0}^{t} v[[i]] d t,\{i, 1, k\}\right] \\
u\left[x_{-}, t_{-}\right]=u_{0}[x] \\
\operatorname{Do}\left[u\left[x_{-}, t_{-}\right]=u[x, t]+u_{i}[x],\{i, 1, k\}\right] \\
u[x, t] \\
a=1 ; b=1 ; c=1 \\
m=2 \\
u[x, t] .
\end{gathered}
$$


The Adomian solution obtained is

$$
\begin{aligned}
& u(x, t) \\
& =1+x+x^{2}+2 t^{2}(1+x(1+x)) \\
& \times\left(24+45 x+185 x^{2}+4 x\left(25+70 x^{2}\right)+4\left(2+25 x^{2}+35 x^{4}\right)\right)+\frac{1}{3} t^{3}(1+x(1+x)) \\
& \times\left(60+1860 x+8 x\left(1860+7995 x^{2}\right)+2\left(570+8370 x^{2}\right)\right. \\
& +8 x\left(1665+10770 x^{2}+12825 x^{4}\right)+4\left(720+14490 x^{2}+29370 x^{4}\right) \\
& \left.+8\left(75+1665 x^{2}+5385 x^{4}+4275 x^{6}\right)\right)+\frac{1}{3} t^{4}(1+x(1+x)) \\
& \times\left(8160+11310 x+180390 x^{2}+4 x\left(50610+318390 x^{2}\right)\right. \\
& +4\left(13380+414000 x^{2}+1172820 x^{4}\right)+4 x\left(160950+1507380 x^{2}+2417550 x^{4}\right) \\
& +16 x\left(20370+222060 x^{2}+585450 x^{4}+429000 x^{6}\right) \\
& +4\left(17970+605070 x^{2}+2705190 x^{4}+2807850 x^{6}\right) \\
& \left.+16\left(600+20370 x^{2}+111030 x^{4}+195150 x^{6}+107250 x^{8}\right)\right)+\frac{1}{60} t^{5}(1+x(1+x)) \\
& \times\left(15120+1275120 x+2\left(486360+16082040 x^{2}\right)\right. \\
& +8 x\left(5172000+45672540 x^{2}\right)+4\left(3226560+145378920 x^{2}+558401640 x^{4}\right) \\
& +8 x\left(36485460+477387720 x^{2}+1007253300 x^{4}\right) \\
& +64 x\left(8126460+123096840 x^{2}+424022700 x^{4}+390053400 x^{6}\right) \\
& +8\left(5708700+279944820 x^{2}+1685856660 x^{4}+2244119100 x^{6}\right) \\
& +32 x\left(5402520+86621760 x^{2}+373392000 x^{4}+591280800 x^{6}+309309000 x^{8}\right) \\
& +16\left(2417760+119127600 x^{2}+868513680 x^{4}+1945018800 x^{6}+1317980400 x^{8}\right) \\
& \left.+32\left(113400+5402520 x^{2}+43310880 x^{4}+124464000 x^{6}+147820200 x^{8}+61861800 x^{10}\right)\right) \\
& +t(1+x(1+x))(2+2(1+5 x(1+x))) .
\end{aligned}
$$

The solutions in Figure 1 increase algebraically as is expected from algebraic behavior of initial condition and the form of $f(u)$.

(ii) $a=b=c=1$ and $m=-2$ (Figure 2). 


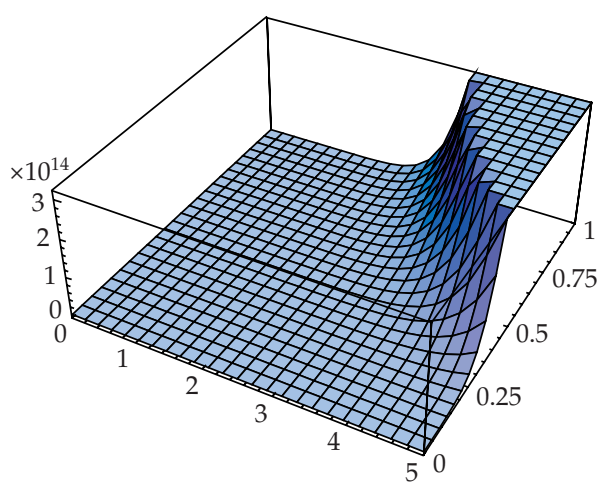

(a)

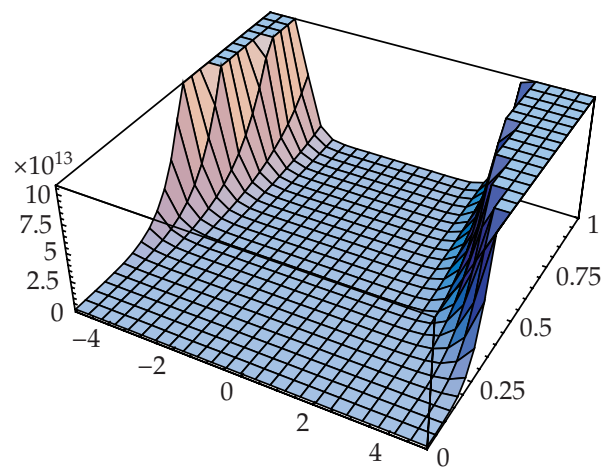

(b)

Figure 1: (a) Graph of Adomian solution for $\{x, 0,5\},\{t, 0,1\}$. (b) Graph Adomian solution for $\{x,-5,5\},\{t, 0,1\}$.

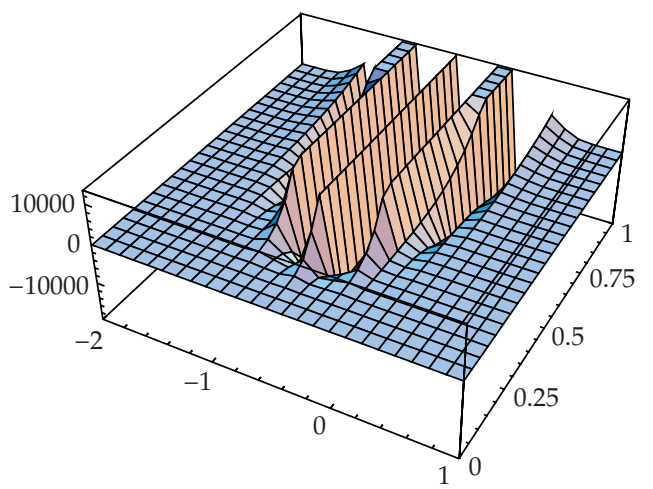

Figure 2: Graph of Adomian solution for the range $\{x,-2,1\},\{t, 0,1\}$.

As the diffusivity in this case is decreasing function of $u$, the solution exhibits the change in the quadratically increasing initial temperature.

(iii) $a=b=c=1$ and $m=1 / 2$ (Figure 3).

Case $B\left(g(x)=e^{-a x^{2}}\right)$. The Adomian solution for general $a, m$ can be obtained from authors as Mathematica file. Some particular cases are considered as follows.

(i) $a=2$ and $m=2$

The Adomian solution is

$$
\begin{aligned}
u(x, t)= & \mathrm{e}^{-2 x^{2}}+4 \mathrm{e}^{-6 x^{2}} t\left(-1+12 x^{2}\right)+8 \mathrm{e}^{-10 x^{2}} t^{2}\left(11-400 x^{2}+1200 x^{4}\right) \\
& +\frac{32}{3} \mathrm{e}^{-14 x^{2}} t^{3}\left(-315+22692 x^{2}-181552 x^{4}+291648 x^{6}\right) \\
& +\frac{32}{3} \mathrm{e}^{-18 x^{2}} t^{4}\left(16425-1947360 x^{2}+28962720 x^{4}-115402752 x^{6}+123607296 x^{8}\right) \\
+ & \frac{128}{15} \mathrm{e}^{-22 x^{2}} t^{5}\left(-1326840+233242200 x^{2}-5491343520 x^{4}+38961513344 x^{6}\right. \\
& \left.\quad-99063148800 x^{8}+78562446336 x^{10}+945\left(-1+20 x^{2}\right)\right) .
\end{aligned}
$$




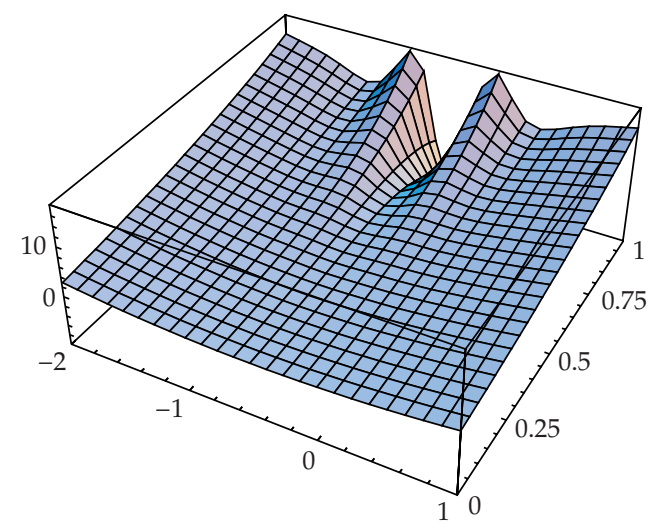

Figure 3: Graph of Adomian solution for the range $\{x,-2,1\},\{t, 0,1\}$.

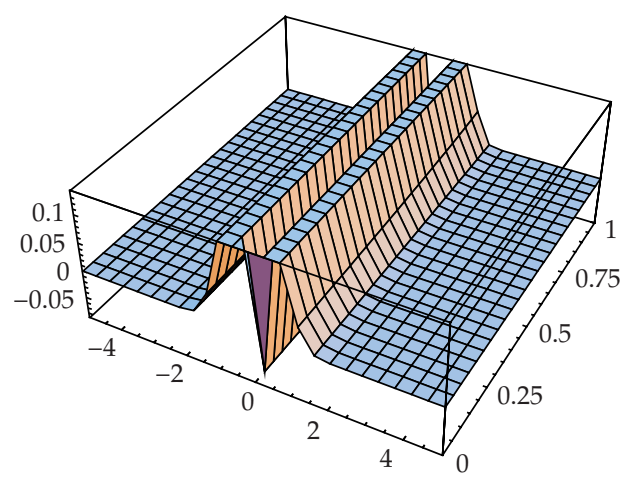

(a)

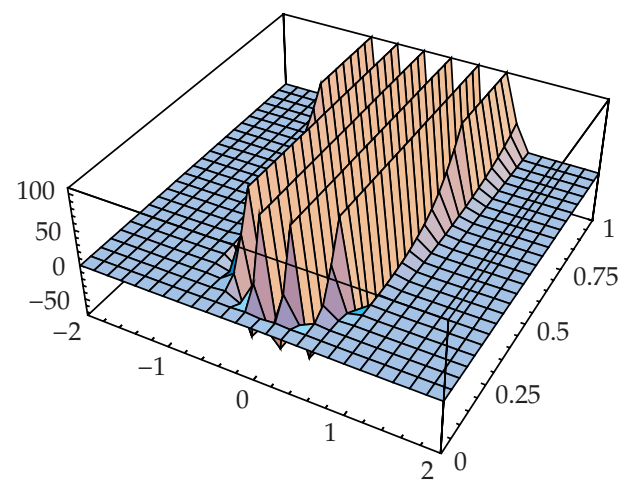

(b)

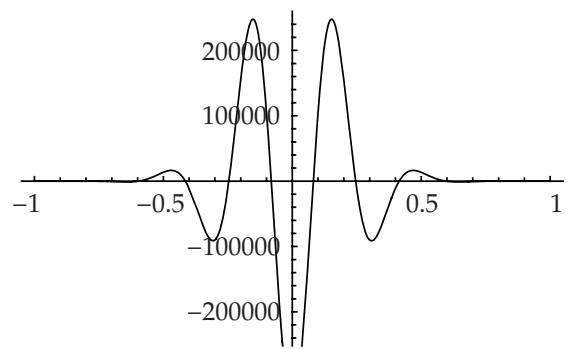

(c)

Figure 4: (a) Graph of solution for the range $\{x,-5,5\},\{t, 0,1\}$. (b) Graph of solution for the range $\{x,-2,2\},\{t, 0,1\}$. (c) Graph for fixed $t=0.5$ for the range $\{x,-1,1\}$.

Figure 4 displays how the bell-shaped initial temperature interacts with quadratic dependence of diffusivity.

(ii) $a=2$ and $m=-2$ (Figure 5). 


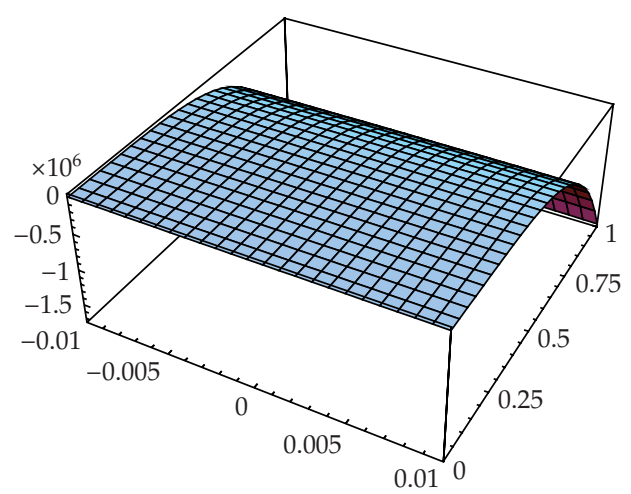

(a)



(b)

Figure 5: (a) Graph for the range $\{x,-.01, .01\},\{t, 0,1\}$. (b) Graph for the range $\{x,-1.5, .01\},\{t, 0,1\}$.

The Adomian solution is

$$
\begin{aligned}
u(x, t)= & \mathrm{e}^{-2 x^{2}}+4 \mathrm{e}^{2 x^{2}} t\left(-1-4 x^{2}\right)+8 \mathrm{e}^{6 x^{2}} t^{2}\left(-5-96 x^{2}-144 x^{4}\right) \\
& +\frac{32}{3} \mathrm{e}^{10 x^{2}} t^{3}\left(-91-4028 x^{2}-19120 x^{4}-17600 x^{6}\right) \\
& +\frac{32}{3} \mathrm{e}^{14 x^{2}} t^{4}\left(-3287-260480 x^{2}-2523104 x^{4}-6375936 x^{6}-4202240 x^{8}\right) \\
& +\frac{128}{15} \mathrm{e}^{18 x^{2}} t^{5}\left(-191704-23954712 x^{2}-390296736 x^{4}-1877037696 x^{6}\right. \\
& \left.-3158528256 x^{8}-1613177856 x^{10}+945\left(-1+20 x^{2}\right)\right) .
\end{aligned}
$$

(iii) $a=2$ and $m=1 / 2$ (Figure 6).

The Adomian solution is

$$
\begin{aligned}
u(x, t)= & \mathrm{e}^{-2 x^{2}}+4\left(\mathrm{e}^{-2 x^{2}}\right)^{3 / 2} t\left(-1+6 x^{2}\right)+8 \mathrm{e}^{-4 x^{2}} t^{2}\left(5-76 x^{2}+96 x^{4}\right) \\
+ & \frac{32}{3}\left(\mathrm{e}^{-2 x^{2}}\right)^{5 / 2} t^{3}\left(-\frac{219}{4}+\frac{3009 x^{2}}{2}-4615 x^{4}+2850 x^{6}\right) \\
+ & \frac{32}{3} \mathrm{e}^{-6 x^{2}} t^{4}\left(\frac{2031}{2}-43365 x^{2}+233406 x^{4}-337716 x^{6}+131760 x^{8}\right) \\
+ & \frac{128}{15}\left(\mathrm{e}^{-2 x^{2}}\right)^{7 / 2} t^{5}\left(-\frac{433389}{16}+\frac{13476579 x^{2}}{8}-\frac{27882147 x^{4}}{2}+34527269 x^{6}\right. \\
& \left.-30761241 x^{8}+8579214 x^{10}+945\left(-1+20 x^{2}\right)\right) .
\end{aligned}
$$




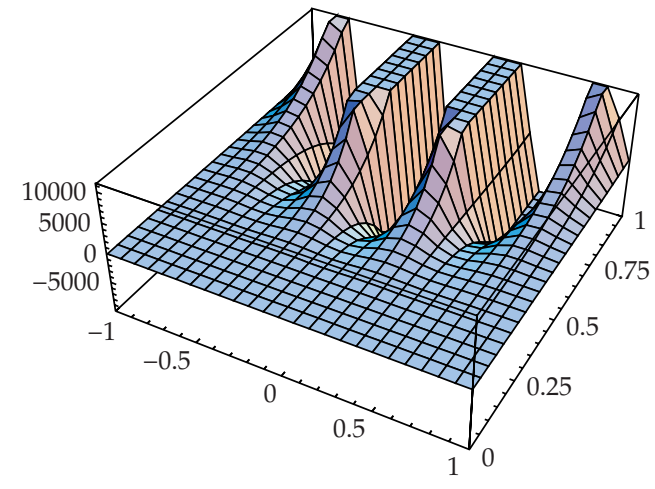

(a)

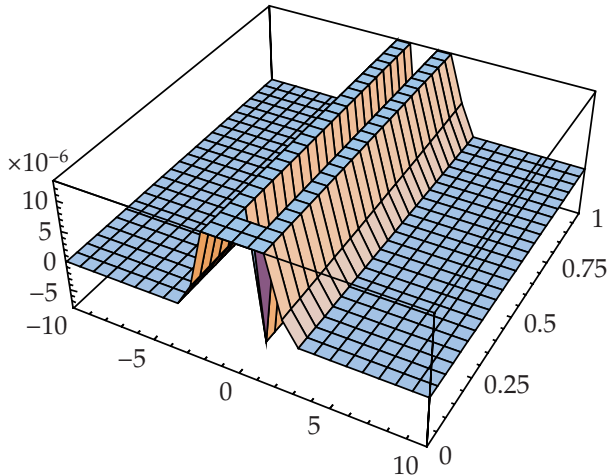

(b)

Figure 6: (a) Graph for the range $\{x,-1,1\},\{t, 0,1\}$. (b) Graph for the range $\{x,-10,10\},\{t, 0,1\}$.

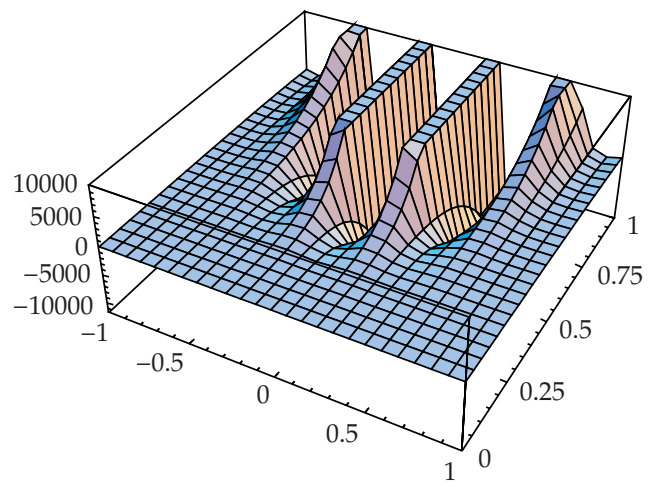

(a)

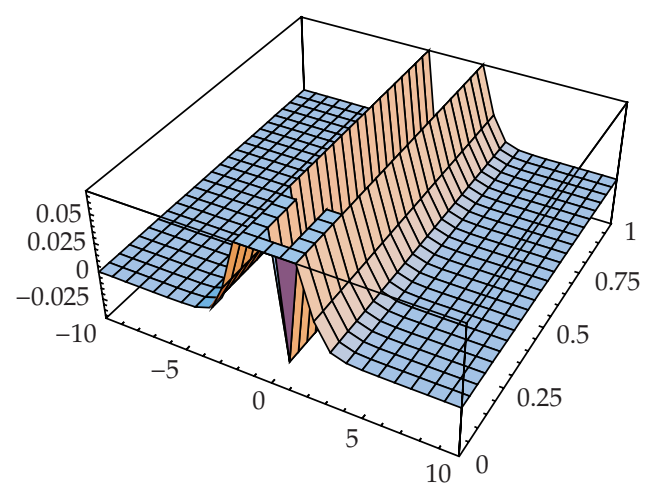

(b)

Figure 7: (a) Graph for the range $\{x,-1,1\},\{t, 0,1\}$. (b) Graph for the range $\{x,-10,10\},\{t, 0,1\}$.

Case $C\left(g(x)=\operatorname{sech}^{2} x\right)$. The Adomian solution for general $m$ can be obtained from authors as Mathematica file. Some particular cases are considered as follows.

(i) $m=2$.

The Adomian solution is

$$
\begin{aligned}
u(x, t) & \\
= & \operatorname{sech}(x)^{2}+2 t(-4+3 \cosh (2 x)) \operatorname{sech}(x)^{8} \\
+ & 3 t^{2}(161-178 \cosh (2 x)+25 \cosh (4 x)) \operatorname{sech}(x)^{14} \\
+ & t^{3}(-54900+71641 \cosh (2 x)-18772 \cosh (4 x)+1519 \cosh (6 x)) \\
\times & \operatorname{sech}(x)^{20}+\frac{1}{4} t^{4}(35318621-50550350 \cosh (2 x)+18047504 \cosh (4 x) \\
& -2916178 \cosh (6 x)+160947 \cosh (8 x)) \\
\times \operatorname{sech}(x)^{26}+\frac{1}{20} t^{5}( & -35893153056+54495231330 \cosh (2 x)-23506173696 \cosh (4 x) \\
& +5488700877 \cosh (6 x)-621401568 \cosh (8 x) \\
& +25573713 \cosh (10 x)) \operatorname{sech}(x)^{32} .
\end{aligned}
$$


Here the initial condition is soliton like. This is reflected in the Figure 7 as the diffusivity varies quadratically.

(ii) $m=-2$ (Figure 8).

The Adomian solution is

$$
\begin{aligned}
u(x, t)= & -2 t \cosh (2 x)-t^{2} \cosh (x)^{2}(1-2 \cosh (2 x)+9 \cosh (4 x)) \\
- & \frac{1}{3} t^{3} \cosh (x)^{4}(-44+85 \cosh (2 x)-76 \cosh (4 x)+275 \cosh (6 x)) \\
- & \frac{1}{12} t^{4} \cosh (x)^{6}(2865-5862 \cosh (2 x)+5968 \cosh (4 x)-5178 \cosh (6 x) \\
& +16415 \cosh (8 x))-\frac{1}{60} t^{5} \cosh (x)^{8} \\
& \times(-303864+606738 \cosh (2 x)-616768 \cosh (4 x)+638373 \cosh (6 x) \\
& -544328 \cosh (8 x)+1575369 \cosh (10 x))+\operatorname{sech}(x)^{2} .
\end{aligned}
$$

(iii) $m=1 / 2$ (Figure 9).

The Adomian solution is

$$
\begin{aligned}
u(x, t)= & \operatorname{sech}(x)^{2}+\frac{3}{2} t^{2}(56-52 \cosh (2 x)+4 \cosh (4 x)) \operatorname{sech}(x)^{8}+\frac{1}{8} t^{4} \\
\times & \left(\frac{5889415}{8}-\frac{3750383}{4} \cosh (2 x)+232028 \cosh (4 x)\right. \\
& \left.\quad-\frac{76417}{4} \cosh (6 x)+\frac{2745}{8} \cosh (8 x)\right) \\
\times & \operatorname{sech}(x)^{14}+2 t\left(-\frac{5}{2}+\frac{3}{2} \cosh (2 x)\right) \operatorname{sech}(x)^{4} \sqrt{\operatorname{sech}(x)^{2}} \\
+ & \frac{1}{2} t^{3}\left(-\frac{37917}{8}+\frac{86005}{16} \cosh (2 x)-\frac{7163}{8} \cosh (4 x)+\frac{475}{16} \cosh (6 x)\right) \\
\times & \operatorname{sech}(x)^{8}\left(\operatorname{sech}(x)^{2}\right)^{3 / 2}+\frac{1}{40} t^{5} \\
\times & \left(-\frac{22986251157}{128}+\frac{31585649589}{128} \cosh (2 x)-\frac{2501116101}{32} \cosh (4 x)\right. \\
& \left.+\frac{2695647273}{256} \cosh (6 x)-\frac{64605399}{128} \cosh (8 x)+\frac{1429869}{256} \cosh (10 x)\right) \\
& \times \operatorname{sech}(x)^{12}\left(\operatorname{sech}(x)^{2}\right)^{5 / 2} \cdot
\end{aligned}
$$




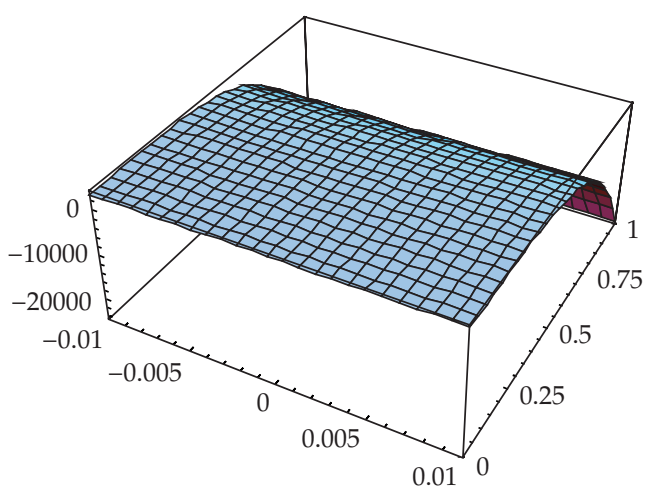

(a)

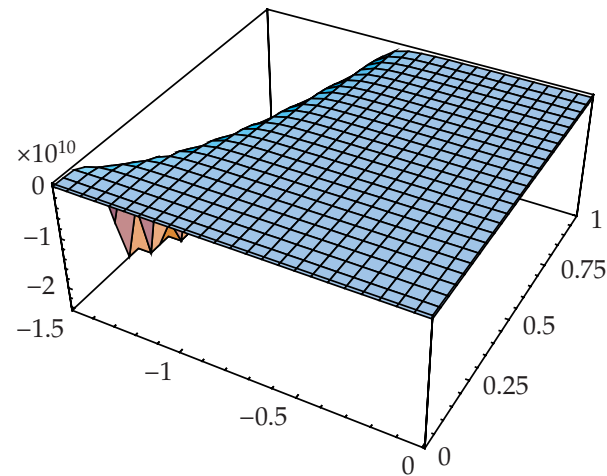

(b)

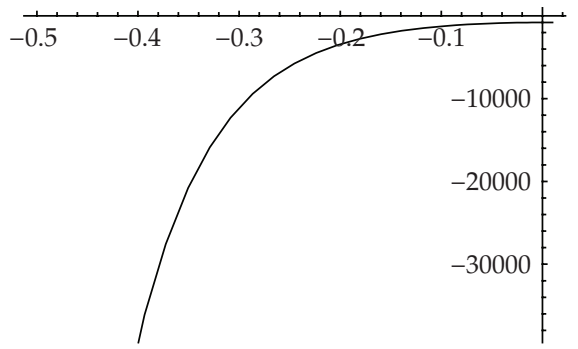

(c)

Figure 8: (a) Graph for the range $\{x,-.01, .01\},\{t, 0,1\}$. (b) Graph for the range $\{x,-1.5, .01\},\{t, 0,1\}$. (c) Graph for fixed $t=0.5$ for the range $\{x,-0.5,0.01\}$.

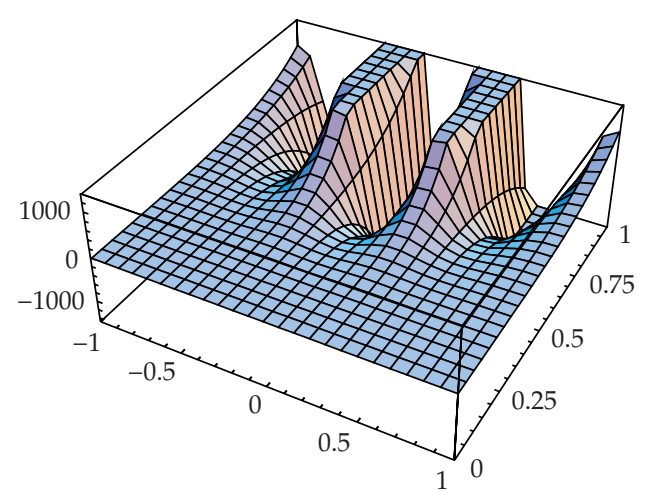

(a)

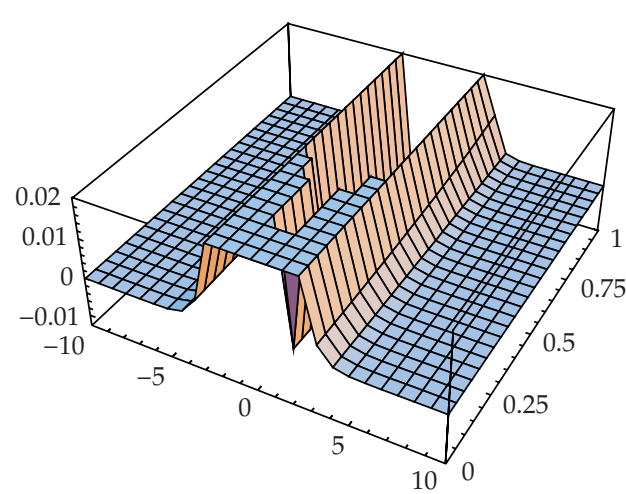

(b)

Figure 9: (a) Graph for the range $\{x,-1,1\},\{t, 0,1\}$. (b) Graph for the range $\{x,-10,10\},\{t, 0,1\}$.

\section{Conclusion}

The Adomian decomposition method has been applied to obtain solutions of the heat equation with power nonlinearity in the diffusivity. The solutions are presented for some typical initial temperature profiles like a quadratic function or or $e^{-a x^{2}}$ or $\operatorname{sech}^{2} x$. The 
interaction of the initial temperature with diffusivity is also discussed for different cases of solutions investigated here.

\section{Acknowledgment}

The authors would like to thank King Fahd University of Petroleum and Minerals, Dhahran, Saudi Arabia, for the support and research facilities provided to complete this work.

\section{References}

[1] M. Necati Ozisk, Heat Conduction, John Wiley \& Sons, New York, NY, USA, 2nd edition, 1993.

[2] G. Adomian and G. E. Adomian, "A global method for solution of complex systems," Mathematical Modelling, vol. 5, no. 4, pp. 251-263, 1984.

[3] G. Adomian, "A new approach to nonlinear partial differential equations," Journal of Mathematical Analysis and Applications, vol. 102, no. 2, pp. 420-434, 1984.

[4] G. Adomian and R. Rach, "Polynomial nonlinearities in differential equations," Journal of Mathematical Analysis and Applications, vol. 109, no. 1, pp. 90-95, 1985.

[5] G. Adomian, "A review of the decomposition method in applied mathematics," Journal of Mathematical Analysis and Applications, vol. 135, no. 2, pp. 501-544, 1988.

[6] G. Adomian, Solving Frontier Problems of Physics: The Decomposition Method, vol. 60 of Fundamental Theories of Physics, Kluwer Academic Publishers, Dordrecht, The Netherlands, 1994.

[7] E. Babolian and Sh. Javadi, "New method for calculating Adomian polynomials," Applied Mathematics and Computation, vol. 153, no. 1, pp. 253-259, 2004.

[8] A.-M. Wazwaz, "A comparison between Adomian decomposition method and Taylor series method in the series solutions," Applied Mathematics and Computation, vol. 97, no. 1, pp. 37-44, 1998.

[9] A.-M. Wazwaz, "A reliable modification of Adomian decomposition method," Applied Mathematics and Computation, vol. 102, no. 1, pp. 77-86, 1999.

[10] A.-M. Wazwaz, "A new algorithm for calculating Adomian polynomials for nonlinear operators," Applied Mathematics and Computation, vol. 111, no. 1, pp. 53-69, 2000.

[11] A.-M. Wazwaz, "Approximate solutions to boundary value problems of higher order by the modified decomposition method," Computers E Mathematics with Applications, vol. 40, no. 6-7, pp. 679-691, 2000.

[12] Y. Cherruault, "Convergence of Adomian's method," Kybernetes of Cybernetics and General Systems, vol. 18, no. 2, pp. 31-38, 1989.

[13] Y. Cherruault and G. Adomian, "Decomposition methods: a new proof of convergence," Mathematical and Computer Modelling, vol. 18, no. 12, pp. 103-106, 1993.

[14] D. Lesnic, "Convergence of Adomian's decomposition method: periodic temperatures," Computers $\mathcal{E}$ Mathematics with Applications, vol. 44, no. 1-2, pp. 13-24, 2002.

[15] C.-H. Chiu and C.-K. Chen, "A decomposition method for solving the convective longitudinal fins with variable thermal conductivity," International Journal of Heat and Mass Transfer, vol. 45, no. 10, pp. 2067-2075, 2002.

[16] L. Dresner, Similarity Solutions of Nonlinear Partial Differential Equations, vol. 88 of Research Notes in Mathematics, Pitman, Boston, Mass, USA, 1983.

[17] E. A. Saied and M. M. Hussein, "New classes of similarity solutions of the inhomogeneous nonlinear diffusion equations," Journal of Physics A, vol. 27, no. 14, pp. 4867-4874, 1994.

[18] E. A. Saied, "The non-classical solution of the inhomogeneous non-linear diffusion equation," Applied Mathematics and Computation, vol. 98, no. 2-3, pp. 103-108, 1999.

[19] A.-M. Wazwaz, "Exact solutions to nonlinear diffusion equations obtained by the decomposition method," Applied Mathematics and Computation, vol. 123, no. 1, pp. 109-122, 2001. 


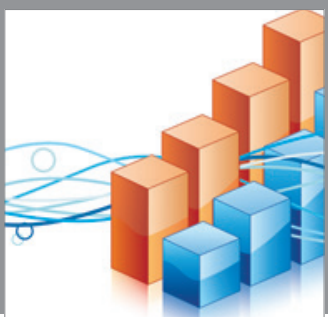

Advances in

Operations Research

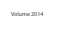

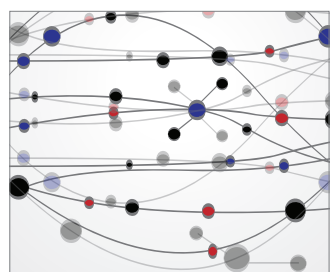

\section{The Scientific} World Journal
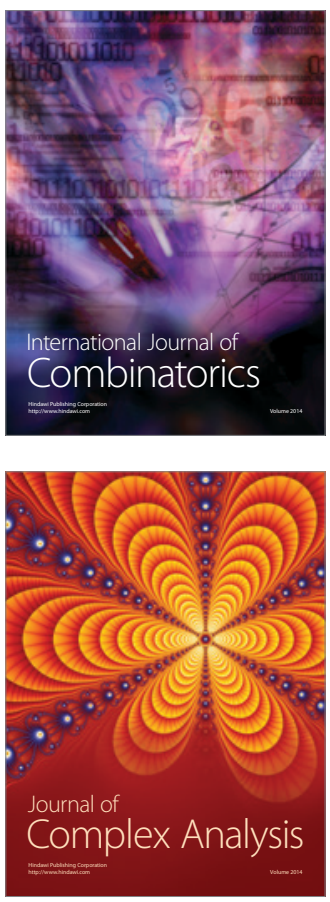

International Journal of

Mathematics and

Mathematical

Sciences


Journal of

Applied Mathematics
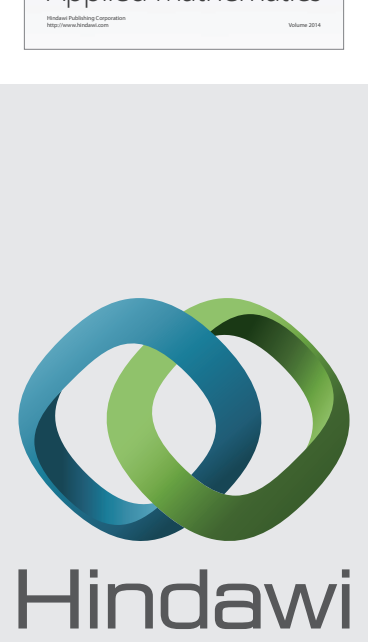

Submit your manuscripts at http://www.hindawi.com
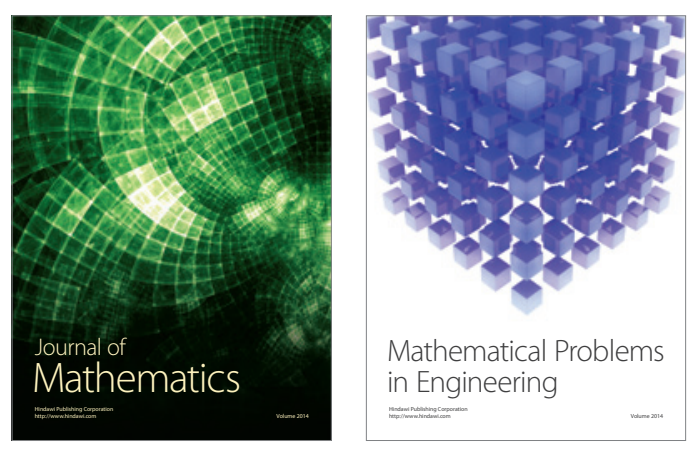

Mathematical Problems in Engineering
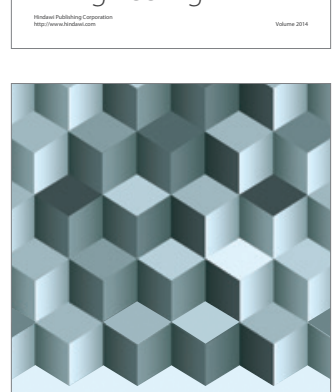

Journal of

Function Spaces


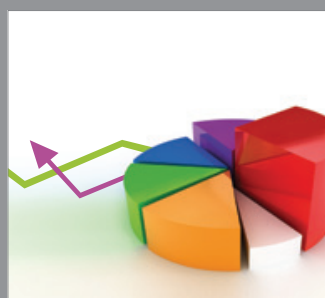

ournal of

Probability and Statistics

Promensencen
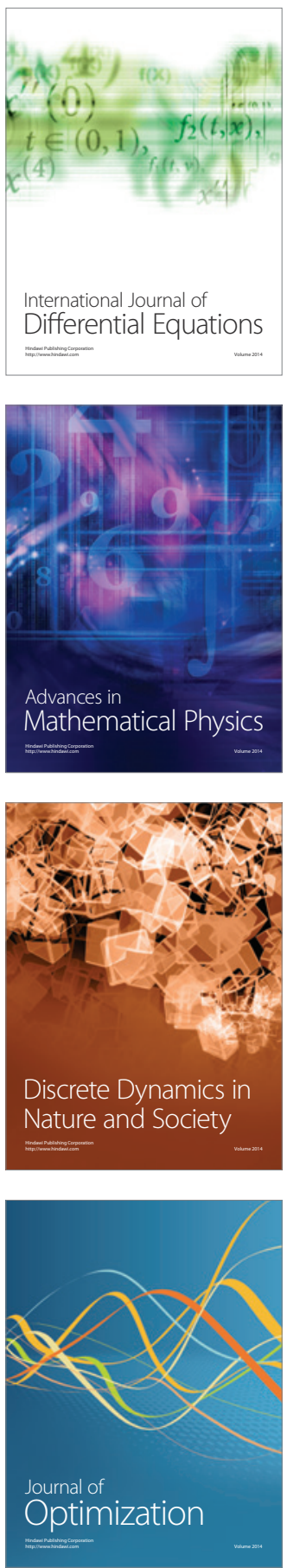